\title{
Molecular Simulation of Fundamental Processes in Nanoparticle - Polymer - Nanoparticle Systems Under Tensile Load
}

\author{
Dirk Zahn
}

\begin{abstract}
We demonstrate molecular modeling of polymer (polyacrylate) association to $\mathrm{ZnO}$ nanoparticles by means of a recently developed molecule-by-molecule association approach. Upon multiple acrylate association steps, potential sites for connecting $\mathrm{ZnO}$ particles are elaborated and explored under tensile loading from molecular dynamics simulation. This offers molecular level insights into processes that account for elastic and plastic deformation, creep and self-healing in $\mathrm{ZnO}$-polymer composite materials.
\end{abstract}

Index Terms-Nanocomposites, mechanical properties, molecular mechanisms.

\section{INTRODUCTION}

Composites of inorganic nanoparticles and polymers currently receive great attention because of its unique mechanical properties. In dental fillings, for example, composites of $\mathrm{ZnO}$ nanoparticles and polymers are applied to provide enamel-like properties [1]. The mechanical properties of teeth generally (and of enamel in particular) encompass two mayor aspects: hardness and resilience. An ideal filling should reflect both properties to optimally replace parts of the tooth surface.

In nature, nanostructured composites of calcium phosphate (apatite) and proteins (amelogenin and/or collagen) are frequently found to provide the desirable mechanical properties of teeth. Human enamel contains about 98 weight- $\%$ of inorganic nanorods (carbonated apatite) which are embedded by a protein-rich matrix. While the former accounts for enamel hardness, resilience is attributed to the composite character resulting from the organic-inorganic interfaces [2]-[4]. The molecular mechanisms that lead to the remarkable resilience of enamel are not fully understood, though molecular dynamics simulations dedicated to biomimetic models of reduced complexity shed some light into fundamental processes occurring under mechanical loading [5]. From this, the organic-inorganic interface was found to facilitate plastic deformation by allowing gradually increasing ionic disorder. Strikingly, this process is reversible, and upon stress release the composite relaxes to the original structure.

Inspired by the success of $\mathrm{ZnO}$-polymer nanocomposites as dental filling materials, here we explore fundamental aspects of this class of in principle rather complex, and

Manuscript received December 29, 2012; revised February 28, 2013.

Dirk Zahn is with the Computer Chemistry Centrum, Naegelsbachstr.25, 91052 Erlangen, Germany (e-mail: dirk.zahn@ chemie.uni-erlangen.de). structurally incompletely described compounds. Along this line, we shall focus on two $\mathrm{ZnO}$ nanoparticles that are connected by polyacrylate molecules. While this is strictly spoken a nanosystem rather than a composite, this simplified model nevertheless should be expected to qualitatively account for processes that trigger the deformation and fracture of the corresponding composite.

\section{Simulation Details}

A scale-up model of a $\mathrm{ZnO} / \mathrm{Zn}(\mathrm{OH})_{2}$ core/shell nanoparticle was prepared by first cutting a hexagonal prism of about $5 \times 5 \mathrm{~nm}$ diameters from the $\mathrm{ZnO}$ crystal structure. To mimic a realistic surface, $\mathrm{O}^{2-}$ ion protonation and charge compensation by $\mathrm{Zn}^{2+}$ deficiencies were carefully considered. This was based on an earlier study of $\mathrm{ZnO}$ nucleation from solution [6], suggesting the absence of exposed $\mathrm{O}^{2-}$ ions at the surface of forming $\mathrm{Zn}_{\mathrm{x}} \mathrm{O}_{\mathrm{y}}(\mathrm{OH})_{\mathrm{z}}$ aggregates as a general building rule. The prepared nanorod model comprises 5422 $\mathrm{Zn}^{2+}, 4860 \mathrm{O}^{2-}$ and $1124 \mathrm{OH}^{-}$ions and exhibits no net charge. The polymer models were chosen as chains of 20 acrylate units, thus resulting in a net charge of -20 per polyacrylate molecule.

Molecular dynamics simulations were performed on the basis of empirical interaction potentials adopted from earlier studies of $\mathrm{ZnO}$ nucleation from solution [6] and bismuth oxide association to polyacrylate [7]. The simulation temperature was fixed to $300 \mathrm{~K}$ and a time-step of $1 \mathrm{fs}$ was applied throughout the molecular dynamics simulations. To avoid truncation artifacts of long-range interaction potentials, explicit Coulomb summation was perfomed.

Tensile loading was implemented by displacing two moieties at constant velocity and exploring the potential energy profile as a function of applied displacement. Because of the limited time scales accessible to molecular dynamics simulations relatively large strain rates were chosen. However, by testing a variety of different strain rates, we ensured that the system is allowed sufficient relaxation time to avoid rate dependencies. This check of convergence applies to both, the deformation mechanism and the underlying energy profile. The insights presented in the following were obtained from production runs using a tensile loading rate of $5 \mathrm{~nm} / \mathrm{ns}$.

\section{RESULTS}

\section{A. Polyacrylate Association to the Nanorod Model.}

The manifold of possible molecular arrangements on the 
surface of the nanoparticle calls for a systematic investigation of putative association sites. Focusing on polyacrylate association to the (001) face of the nanorod, we employed the Kawska-Zahn method for searching association sites and exploring subsequent relaxation of the overall system. While more details are described in ref. [5], the key concept is given by placing molecules at random orientation and random position above the nanoparticle surface, and exploring relaxation from molecular dynamics simulation. This procedure was applied iteratively, thus adding surfactant molecules one-by-one until electrostatic repulsion prohibits further association. Despite the -20 net charge per molecule, the (001) surface of $\sim 5 \mathrm{~nm}$ diameter can accommodate up to three polyacrylate molecules. While the first two molecules were found to form $-\mathrm{CO}_{2}^{-} \cdot \cdot \mathrm{Zn}^{2+}$ salt bridges using all acrylate units, the third polyacrylate molecule can only partially arrange in such manner. Further attempts to dock polyacrylate molecules were either rejected to lead to lateral association (Fig. 1).

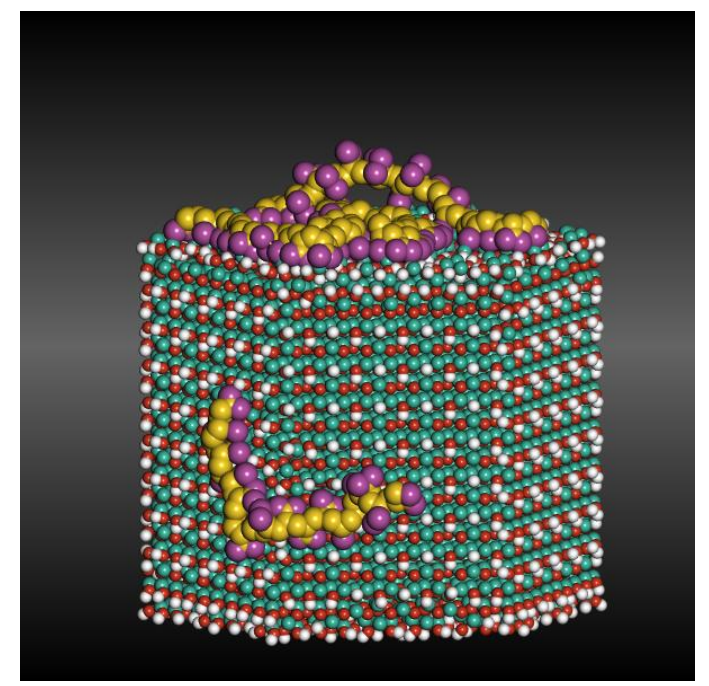

Fig. 1. Illustration of a $\mathrm{ZnO} / \mathrm{Zn}(\mathrm{OH})_{2}$ core/shell nanoparticle which (001) was covered by 3 polyacrylate molecules. Further polyacrylate molecules are rejected or redirected to lateral faces.

\section{B. Nanorod-Polymer-Nanorod Simulations}

Upon covering the nanoparticle surface with a maximum number of polyacrylate molecules we observed sequences of exposed acrylate monomers, i.e. exhibiting $-\mathrm{CO}_{2}^{-}$units that can serve as binding sites for the association of another $\mathrm{ZnO}$ nanoparticle. By placing a replica of our nanorod model above the $\mathrm{ZnO}$-polymer complex we indeed obtained a stable system comprising two nanoparticles that are 'glued' by polyacrylate molecules.

Even for such a small system of two nanoparticles there is an immense variety of possible arrangements. Rather than scanning the full statistics of this exponentially increasing manifold, here we shall elaborate more qualitative insights by the example of a single, arbitrarily chosen $\mathrm{ZnO}$-polymer-ZnO nanosystem. Our model reflects two nanorod of equal size and orientation which are connected by 3 polyacrylate molecules (each of 20 monomeric units). The relaxed nanosystem prepared in such way is illustrated in Fig. 2.

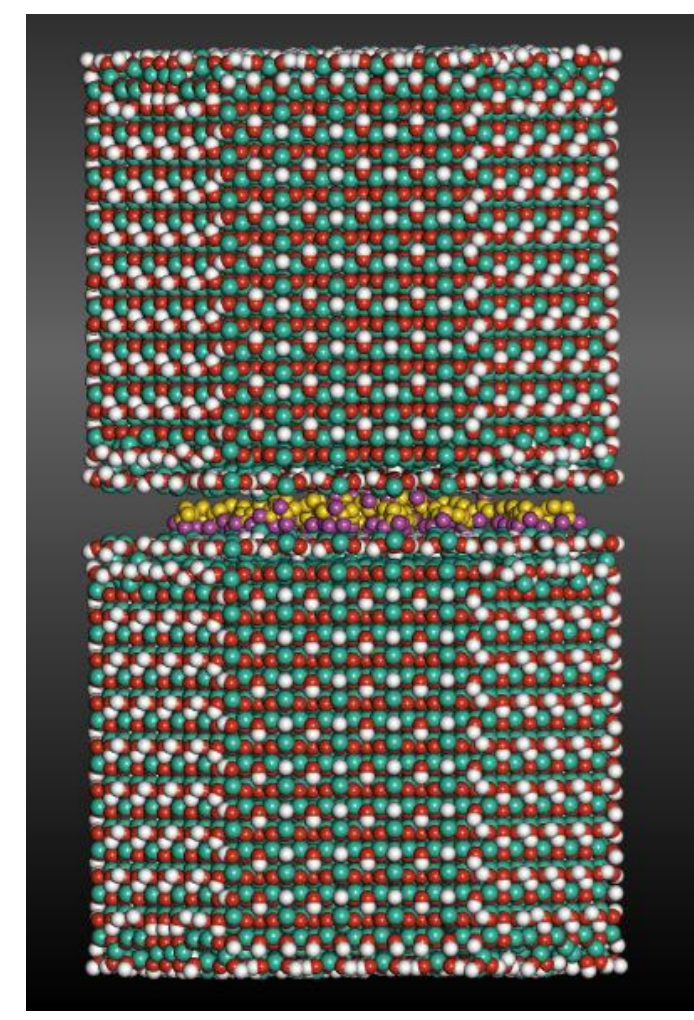

Fig. 2. Model system comprising nanoparticles which (001) faces are 'glued' by 3 polyacrylate molecules.

Despite the electrostatic repulsion of the three polyacrylate molecules of -20 charge, the overall nanosystem was found as quite stable. Deformation and eventual dissociation requires a considerable amount of energy. The underlying energy profile as shown in Fig. 3 may be devised in three characteristic regimes. Upon pulling by up to about $\mathrm{s}=0.5$ $\mathrm{nm}$ the nanosystem remains within the elastic regime (potential energy proportional to $\mathrm{s}^{2}$ ). Elasticity is practically purely related to the deformation of the polyacrylate molecules and the stretching of $-\mathrm{CO}_{2}^{-} \cdot \cdot \mathrm{Zn}^{2+}$ salt bridges.

Within nanoparticle displacement s between 0.5 and 2.5 $\mathrm{nm}$ we observed a linear increase in potential energy. Such constant stress-strain behavior is indicative for creep in $\mathrm{ZnO}$-polymer composites, and, from a qualitative viewpoint our simple model system allows the characterization of the underlying molecular mechanisms. The mechanism of plastic deformation and eventual fracture is illustrated in Fig. 4. Upon increasing strain $\mathrm{s}$, the $-\mathrm{CO}_{2}^{-} \cdot \cdot \mathrm{Zn}^{2+}$ salt bridges are overstretched. The applied pulling rate is sufficiently low to allow bond dissociation to occur sequentially, thus gradually releasing the mechanical stress of the nanoparticle-polymer interface. Strikingly, this process is reversible and the nanosystem elongated by up to $3 \mathrm{~nm}$ can fully 'recover' when tensile load is removed. In the course of relaxation in absence of pulling forces or constraints on the nanoparticle positions, new $-\mathrm{CO}_{2}^{-} \cdot \cdot \mathrm{Zn}^{2+}$ salt bridges are formed giving rise to an analogously 'glued' system of two nanorods. While the newly formed bonds are not necessarily identical to the previously broken ones, we found them as equivalent in terms of the mechanical properties. Indeed, repeating the loading after relaxation from under-critical deformation ( $\mathrm{s}<$ $3 \mathrm{~nm}$ ) lead to practically identical energy profiles. 


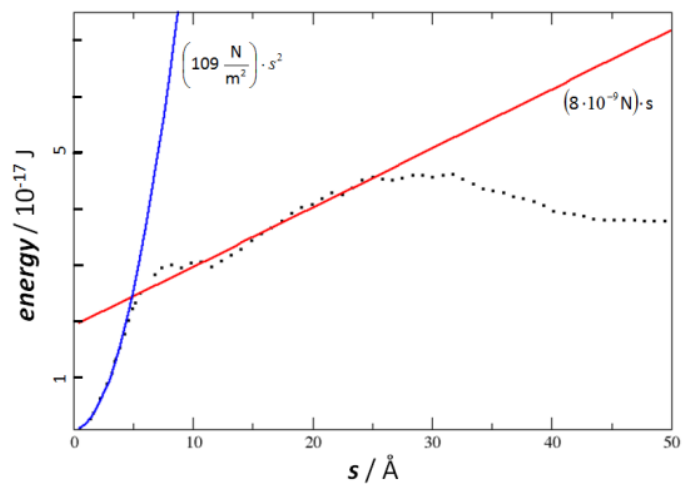

Fig. 3. Potential energy as a function of system elongation from the equilibrium structure. The blue and red curves correspond to quadratic and linear fits to the elastic and creep regime, respectively.

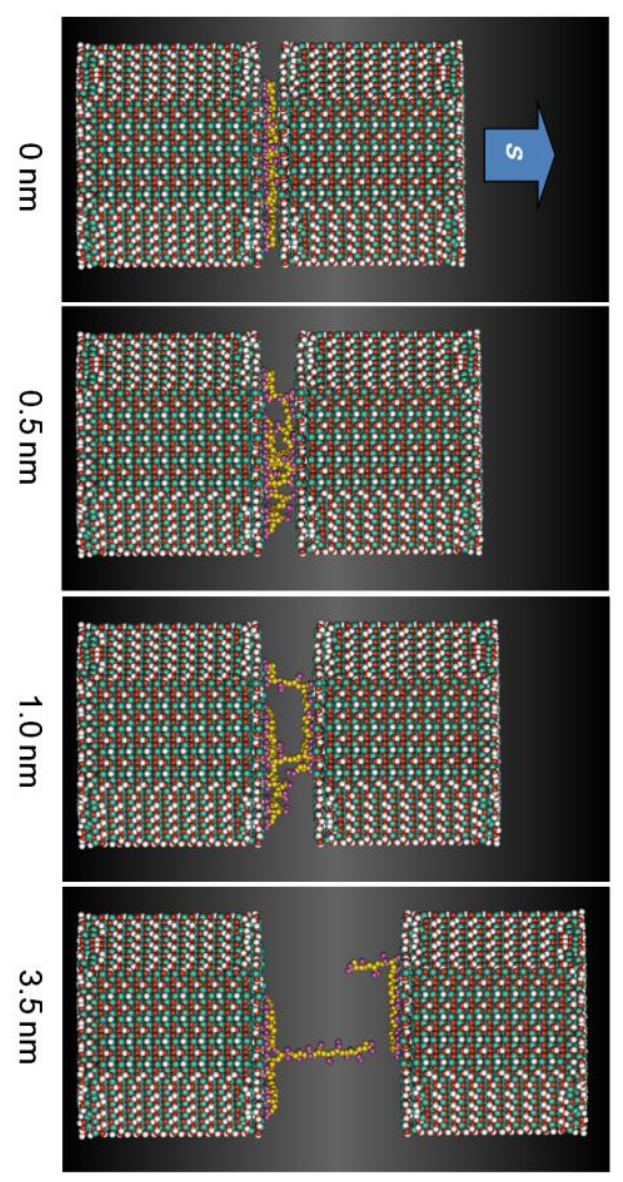

Fig. 4. Nanorod-polyacrylate-nanorod model under tensile load. Elastic deformation by elongation of electrostatic bonds is observed up to $s=0.5$ $\mathrm{nm}$. Further pulling leads to subsequent dissociation of acrylate $\cdot \mathrm{Zn}^{2+}$ bonds until eventual fracture is observed at $s=3.5 \mathrm{~nm}$. Within the $(0.5 \mathrm{~nm}<\mathrm{s}<3$ $\mathrm{nm}$ ) regime the system exhibits a roughly constant restoring force. When releasing tensile load after elongation by $3 \mathrm{~nm}$ or less, reformation of electrostatic bonds leads to full recovery of the system.

\section{CONCLUSION}

A simple model system comprising only two nanoparticles that are connected by a few polymer molecules was demonstrated to suffice as a proxy to study complex mechanical behavior including creep and self-healing. The observed mechanism to sacrifice electrostatic bonds upon load beyond the elastic limit and self-healing after under-critical deformation by reformation of bonds appears quite transferrable and we suggest our findings as a rationale of the deformation characteristics observed for bulk $\mathrm{ZnO}$ nanoparticle - polyacrylate composites.

\section{REFERENCES}

[1] J. R. Davis (Ed.), Handbook of medical devices, Materials Park, U.S.A., ASM International, ch. 2, pp. 283-315, 2003.

[2] L. H. He and M. V. Swain, J. Dentistry, vol. 35, pp. 431-437, 2007.

[3] L. H. He and M. V. Swain, Appl. Phys. Lett., vol. 90, pp. 171916-4, 2007.

[4] L. H. He and M. V. Swain, J. Biomed. Mater. Res. Part A, vol. 91, pp. 352-359, 2008.

[5] D. Zahn, Angew. Chem. Int. Ed., vol. 49, pp. 9405-9407, 2010.

[6] A. Kawska, P. Duchstein, O. Hochrein, and D. Zahn, Nanoletters, 8, pp. 2336-2340, 2008.

[7] L. Miersch, T. Rueffer, H. Lang, S. Schulze, M. Hietschold, D. Zahn, and M. Mehring, Eur. J. Inorg. Chem., vol. 30, pp. 4763-4769, 2010.

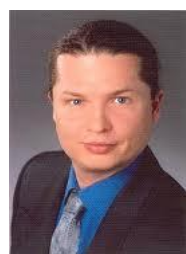

simulation.
Dirk Zahn is a professor of theoretical chemistry at the Computer Chemistry Center of the university of Erlangen-Nuremberg in Germany. Before moving to Erlangen, he worked at Max-Planck institutes in Dresden and Stuttgart and at the ETH Zurich. During his career he was granted $\mathrm{phD}$, postdoc and a Heisenberg-fellowship. $\mathrm{He}$ authored about one hundred peer-reviewed publications in the field of molecular modeling and

Prof. Dr. Zahn is local chair of the German society of chemists and member of the German physical society. 